\title{
The successful application of pyrotinib in the treatment of primary trastuzumab-resistant HER-2-positive breast cancer with bilateral axillary lymph node metastasis: a case report
}

\author{
Yuan Yuan ${ }^{1} \wedge$, Sainan $\mathrm{Hu}^{1}$, Yu Qian ${ }^{2}$, Lili Zhang ${ }^{1 \wedge}$ \\ ${ }^{1}$ Department of Chemotherapy, Jiangsu Cancer Hospital \&Jiangsu Institute of Cancer Research \& The Affiliated Cancer Hospital of Nanjing Medical \\ University, Nanjing China; ${ }^{2}$ Department of Oncology, The Affiliated Cancer Hospital of Nanjing Medical University, Nanjing, China \\ Correspondence to: Lili Zhang. No. 42 Baiziting, Nanjing 210009, China. Email: longrenyu@sina.com.
}

\begin{abstract}
Breast cancer patients with synchronous contralateral axillary lymph node metastasis (CAM) are very rare, and there is a lack of published treatment guidelines for this kind of patients. We presented a human epidermal growth factor receptor 2 (HER-2)-positive breast cancer case, who was diagnosed as CAM with primary trastuzumab resistance. In this case report, we describes a 47-year-old woman diagnosed with HER-2 positive breast cancer with regional lymph nodes metastasis. However, physical examination identified contralateral axillary lymph nodes, the auxiliary inspection did not assist in determining the nature of the right axillary lymph nodes, and there was no obvious mass in the right breast. Hence, we performed the core needle biopsy on the right axillary lymph node, which revealed the presence of metastatic breast adenocarcinoma. The patient received trastuzumab-based treatment, but this only afforded a progressionfree survival of 5 months, owing to primary trastuzumab resistance. She was then successfully treated with pyrotinib, and the outcome was close to clinical complete response (CCR) with a progression-free survival of over 27 months thus far. This case report may help inform clinicians in the diagnosis of breast cancer with CAM and offer the treatment options in HER-2-positive metastasis breast cancer with primary trastuzumab resistance.
\end{abstract}

Keywords: Pyrotinib; primary trastuzumab resistance; contralateral axillary lymph node metastasis (CAM); breast cancer; case report

Submitted May 10, 2021. Accepted for publication Jun 16, 2021.

doi: 10.21037/apm-21-1364

View this article at: https://dx.doi.org/10.21037/apm-21-1364

\section{Introduction}

Breast cancer patients with contralateral axillary lymph node metastasis (CAM) is uncommon, with the incidence rate being $0.8-1.9 \%$ (1). According to the guideline of National Comprehensive Cancer Network (NCCN), CAM is considered a distant metastasis, as it is not a regional draining basin of the breast. At present, there is a lack of evidence and expert consensus on the principle of diagnosis and treatment for patients with bilateral axillary lymph node metastasis, especially with the absence of metastatic disease elsewhere. This report is about a patient with HER-2 positive breast cancer which was refractory to trastuzumab primary resistancetreatment, and at the same time the patient was diagnosed with bilateral axillary lymph node metastasis. Immunohistochemistry of the nodule located in the left breast and ipsilateral axillary lymph node identified the following features: estrogen-receptor negative (ER-), progesterone-receptor $10 \%$ positive $(\mathrm{PR}+10 \%)$, human epidermal growth factor receptor 2 (HER-2) 3+, $\mathrm{Ki}-67+80 \%$, and androgen receptor $90 \%$ positive $(\mathrm{AR}+$ $90 \%)$. Needle puncture biopsy of the left supraclavicular

^ ORCID: Yuan Yuan, 0000-0002-2060-5969; Yu Qian, 0000-0002-6348-9638; Lili Zhang, 0000-0002-4646-6565. 
lymph node showed poorly differentiated carcinoma, likely metastasized from differentiated carcinoma. Immunohistochemistry of the right axillary lymph node indicated the following: ER-, PR-, HER-2 3+, GATA- 3+, and Ki67 $+40 \%$. Cumulatively, the examinations confirmed a diagnosis (in June 2014) of carcinoma of the left breast with left supraclavicular and bilateral axillary lymph node metastasis, stage IV cT2N3M1 (American Joint Committee on Cancer, AJCC 7th edition). The incidence of such patients is low, and there is currently no diagnosis and treatment standard. This article hopes to provide diagnosis and treatment strategies for patients with similar conditions.

Pyrotinib is a small molecule that can irreversibly inhibit pan-ErbB tyrosine kinase with activity against HER-1, HER-2, and HER-4 (2,3), which has been approved by the China Food and Drug Administration (CFDA) for the treatment of HER-2-positive metastatic breast cancer (MBC) (4). Here, we report the diagnostic procedures and clinical management of a patient with HER-2-positive MBC who presented with bilateral axillary lymph node metastasis and primary trastuzumab resistance, emphasizing the successful management using pyrotinib in the case of trastuzumab resistance. We present the following article in accordance with the CARE reporting checklist (available at https://dx.doi.org/10.21037/apm-21-1364).

\section{Case presentation}

A 47-year-old multiparous woman with no medical comorbidities was admitted due to a progressively enlarged mass detected in her left breast. The mass had been increasing in size over the past 3 months. Physical examination identified a hard mass in the left breast measuring approximately $4 \times 3 \mathrm{~cm}^{2}$ located in the lowerinner quadrant region. Meanwhile, the bilateral enlarged axillary lymph nodes were palpable, with fused lymph nodes in the ipsilateral axilla $4 \mathrm{~cm} \times 4 \mathrm{~cm}$ in size and contralateral axillary lymph nodes approximately $1.5 \mathrm{~cm}$ in diameter. Furthermore, there was an enlarged, palpable lymph node at about $1 \mathrm{~cm}$ in diameter in the ipsilateral supraclavicular lymph node region, and the right breast was normal by physical examination.

Mammography, ultrasound evaluation, and breast magnetic resonance imaging (MRI) were further applied to evaluate the patient. The mass detected in the in left internal mammary quadrant was considered malignant and categorized as Breast Imaging-Reporting and Data System (BI-RADS) 4C, with bilateral lymph node enlargement in the axillae also being confirmed. The size of bilateral axillary lymph nodes was $3.18 \mathrm{~cm} \times 1.93 \mathrm{~cm}$ on the left and $1.13 \mathrm{~cm} \times 1.01 \mathrm{~cm}$ on the right; however, there was no obvious mass in the right breast. This suggested that the right breast was normal and excluded the possibility of a contralateral primary tumor (Figure 1A).

To further evaluate the systemic disease, computed tomography (CT) of the brain, neck, chest, abdomen, and bone was performed. Bilateral axillary lymph node enhancement was detected, and no other distant metastases were identified (Figure 1B). A bone scan was performed and also did not detect bone metastases.

Core needle biopsies of the nodule located in the left breast and ipsilateral axillary lymph node revealed invasive breast ductal carcinoma with grade II, axillary lymphatic metastasis of left breast cancer. Immunohistochemistry of the tumor identified the following features: estrogen-receptor negative (ER-), progesteronereceptor $10 \%$ positive ( $\mathrm{PR}+10 \%)$, HER-2 $3+, \mathrm{Ki}-67+80 \%$, and androgen receptor $90 \%$ positive (AR+ $90 \%)$. Needle puncture biopsy of the left supraclavicular lymph node showed poorly differentiated carcinoma, likely metastasized from differentiated carcinoma. To determine whether the contralateral lymph nodes were related to metastasis from the mass located in the left breast, we conducted positron emission tomography-CT (PET-CT) examination, as seen in Figure 1C. It indicated a mass in the lower-inner quadrant region of left breast with a maximum of standard uptake value (SUV) was 10.29 and an SUV of the ipsilateral axillary lymph node was 6.52 . There were enlarged lymph nodes in the right axillary area with a maximum of SUV of 2.30, but this could not exclude malignant tumor metastasis. Hence, we performed core needle biopsy on the right axillary lymph node. The pathological examination revealed metastatic adenocarcinoma. Immunohistochemistry indicated the following: ER-, PR-, HER-2 3+, GATA- 3+, and Ki67 $+40 \%$. Cumulatively, the examinations confirmed a diagnosis (in June 2014) of carcinoma of the left breast with left supraclavicular and bilateral axillary lymph node metastasis, stage IV cT2N3M1 (American Joint Committee on Cancer, AJCC 7th edition).

The patient went through treatments with epirubicin and cyclophosphamide (EC; epirubicin $90 \mathrm{mg} / \mathrm{m}^{2}+$ cyclophosphamide $600 \mathrm{mg} / \mathrm{m}^{2}$ ) for 4 cycles followed by docetaxel and trastuzumab (TH docetaxel $100 \mathrm{mg} / \mathrm{m}^{2}$ and trastuzumab $8 \mathrm{mg} / \mathrm{kg}$ at first dose followed by $6 \mathrm{mg} / \mathrm{m}^{2}$ $\mathrm{q} 3 \mathrm{w}$ ) for 4 cycles. We then selected endocrine therapy combined with trastuzumab as the maintenance treatment. 

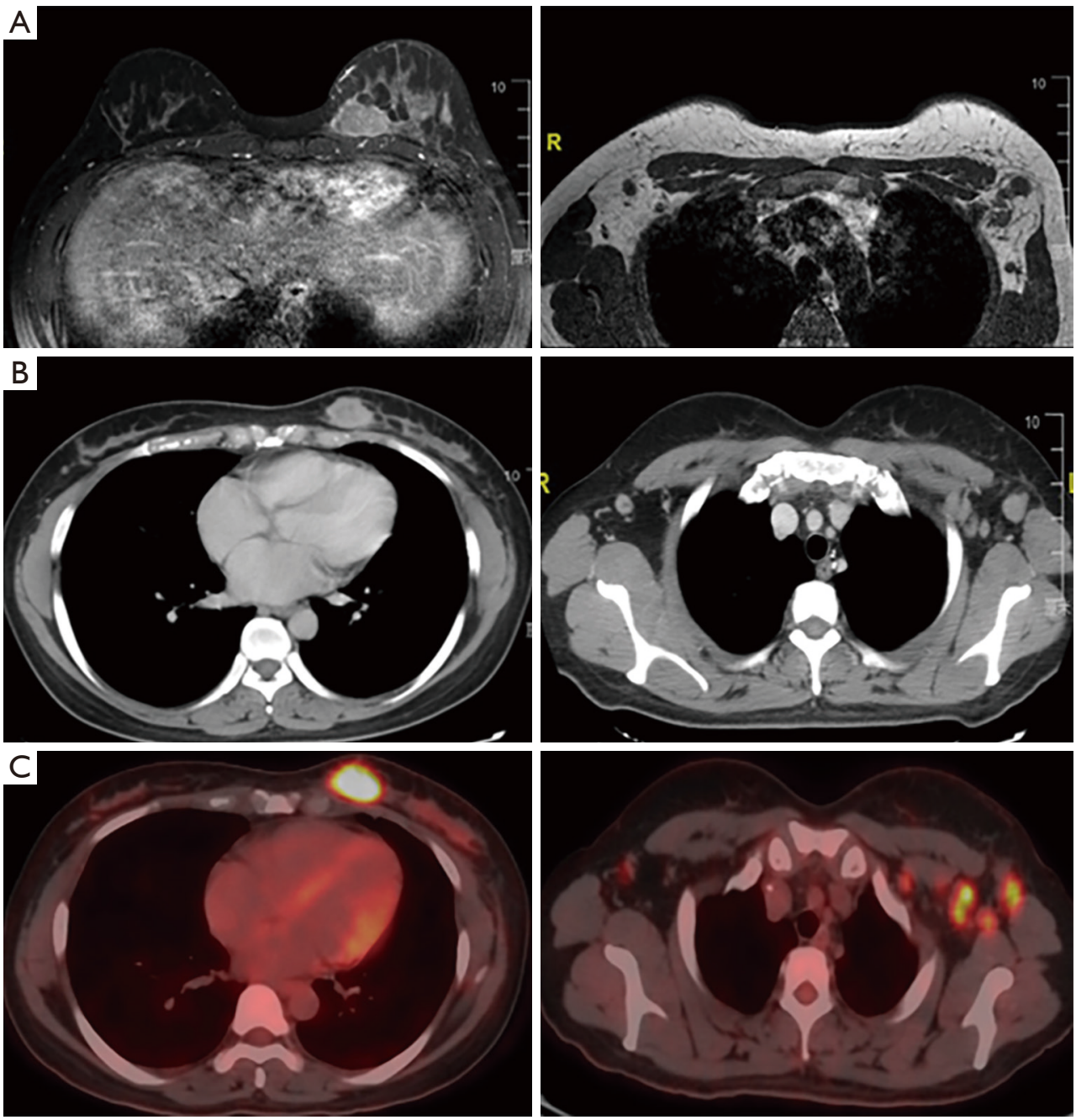

Figure 1 The images of breast and axillary lymph nodes. MRI (A), CT (B) and PET-CT (C) showed a mass in the left breast and enlarged bilateral axillary lymph nodes. MRI, magnetic resonance imaging; CT, computed tomography; PET-CT, positron emission tomographycomputed tomography.

As the patient was premenopausal, the endocrine therapy regimen chosen was ovarian function suppression (OFS) combined with letrozole. CT and MRI showed the efficacy of the treatment with EC followed by $\mathrm{TH}$ as shown in Figure 2.

In December 2018, the patient was evaluated with a CT scan after treatment for 3 months. CT scan showed that the mass in the left breast and ipsilateral axillary lymph node had increased in size. Similar findings were identified by MRI. The evaluation the efficacy was changed to progressive disease (PD; Figure 3); the progressionfree survival (PFS) was 8 months and only 5 months under trastuzumab.
The patient was then administered pyrotinib at $400 \mathrm{mg}$ per day and capecitabine at $1,000 \mathrm{mg} / \mathrm{m}^{2}$ twice a day for 2 weeks from December 2018 to the most recent followup in March 2021. A full-body CT scan showed no evident mass on the left breast, the size of the ipsilateral axillary lymph nodes significantly smaller than previously shown, all the lymph nodes were smaller than $1 \mathrm{~cm}$ in diameter. The whole-body CT and breast MRI were performed every two treatment cycles to evaluate the efficacy during the first year of starting pyrotinib combined with capecitabine, and every 3-4 cyclesweeks in the second year. The outcome was close to clinical complete response (CCR) as demonstrated in Figure 4. 
Baseline
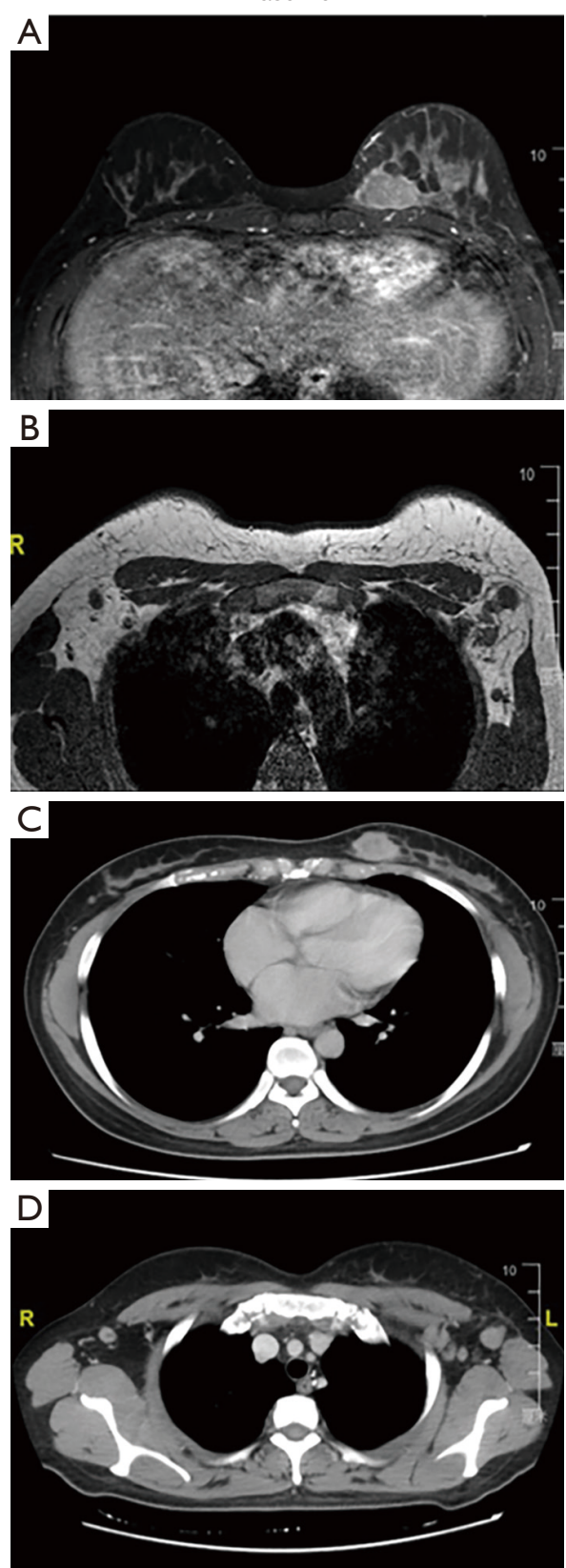

$\mathrm{EC} \times 4$ cycle
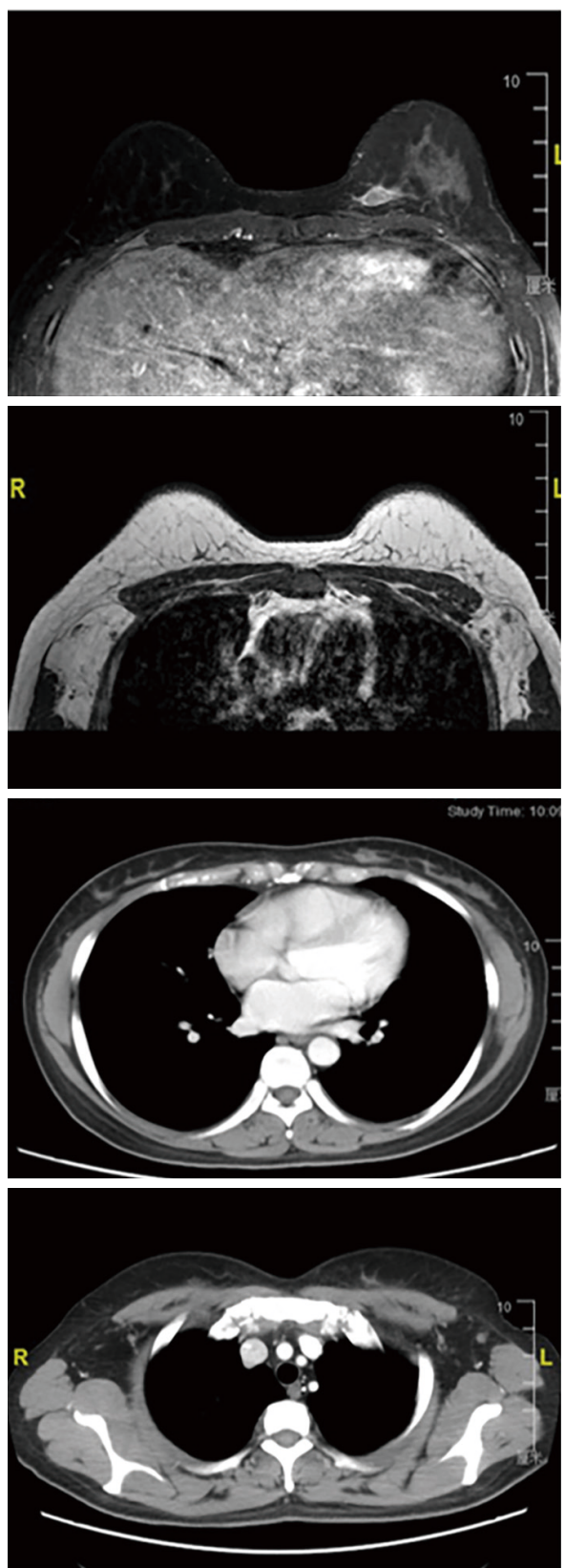

$\mathrm{TH} \times 4$ cycle
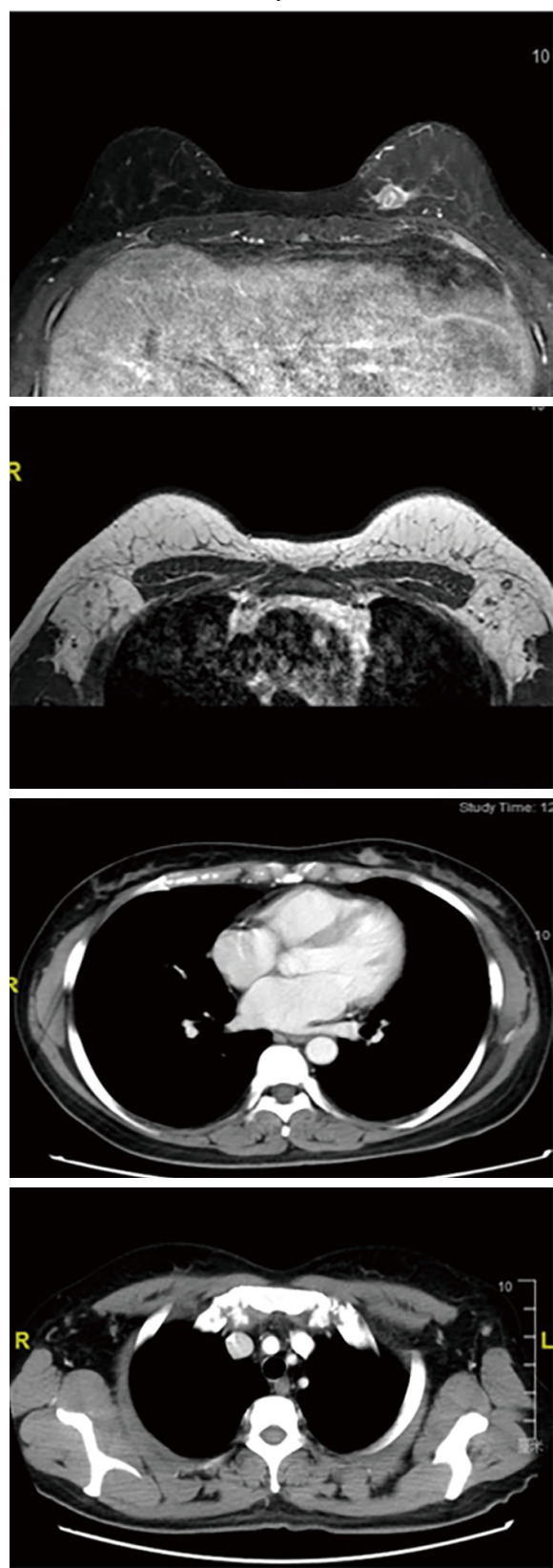

Figure 2 MRI (A,B) and CT (C,D) showing the efficacy of the treatment with EC followed by TH. CT, computed tomography; MRI, magnetic resonance imaging; EC, epirubicin and cyclophosphamide; TH, docetaxel and trastuzumab.

The side effects were well tolerated and manageable. During the first 2 cycles, the main side effects were grade 2 gastrointestinal reactions, including diarrhea, nausea, and vomiting, which were mitigated by symptomatic management with loperamide, mosapride, and omeprazole. We have performed blood routine, biochemical and physical examinations before each cycle of treatments to monitor any potential side effects and safety of the treatments. At the most recent follow-up, the patient no longer had any associated gastrointestinal reaction. The other side effect was grade 1 hand-foot syndrome, which was improved after traditional Chinese medicine therapy. 
2018.9
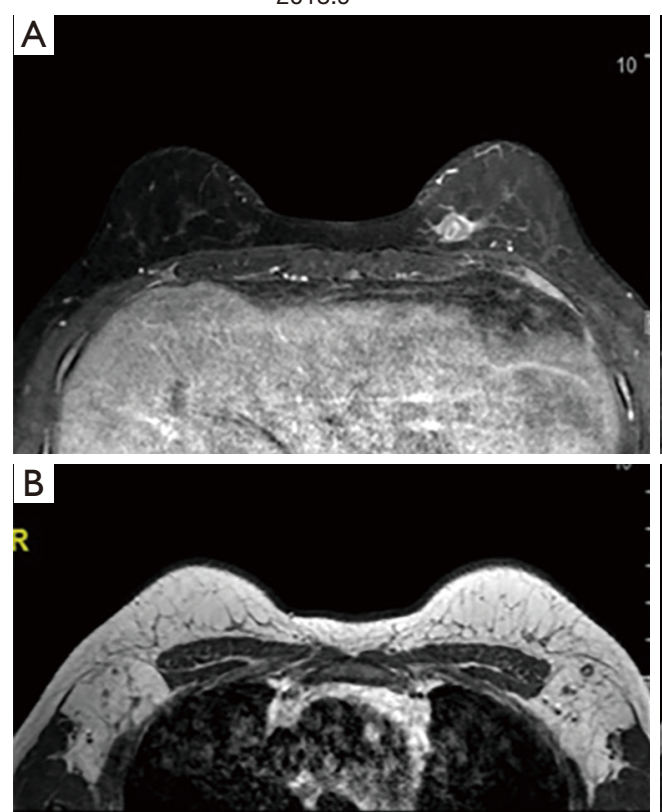

C

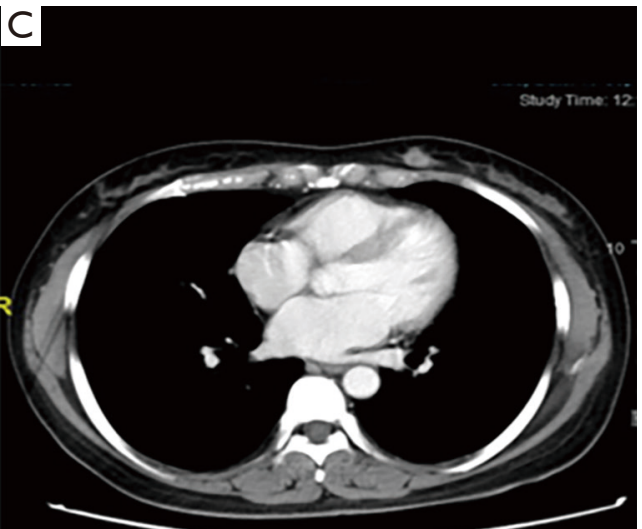

D

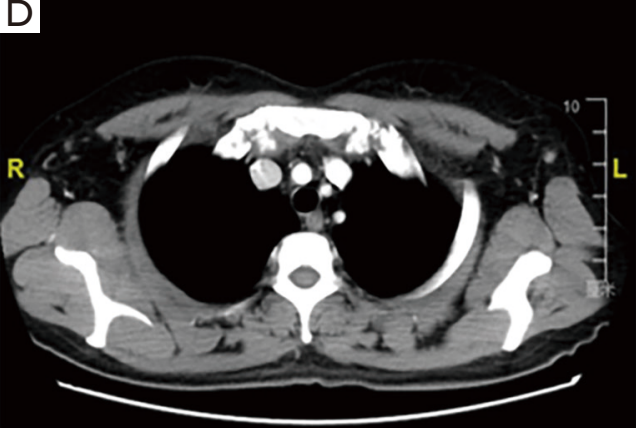

2018.12
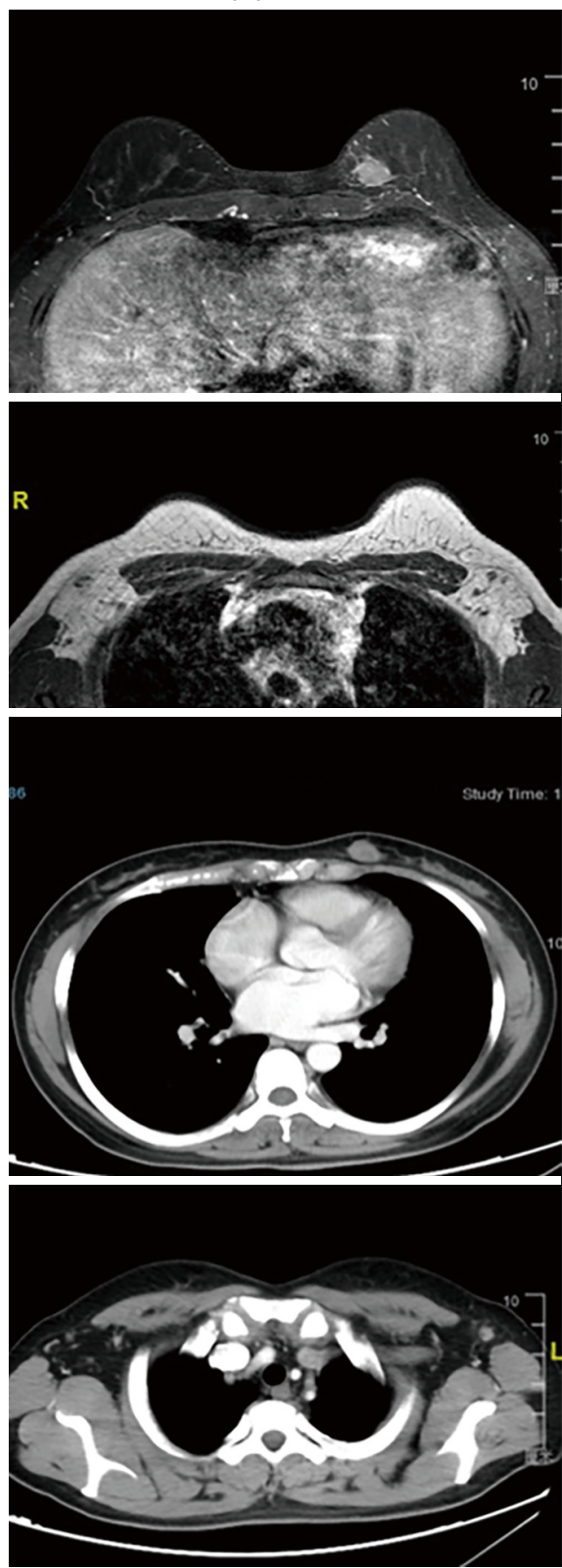

Figure 3 Progression of disease after maintenance treatment by MRI (A,B) and CT (C,D). MRI, magnetic resonance imaging; CT, computed tomography.

All procedures performed in studies involving human participants were in accordance with the ethical standards of the institutional and/or national research committee(s) and with the Helsinki Declaration (as revised in 2013). Written informed consent was obtained from the patient for publication of this manuscript and any accompanying 
2018.12
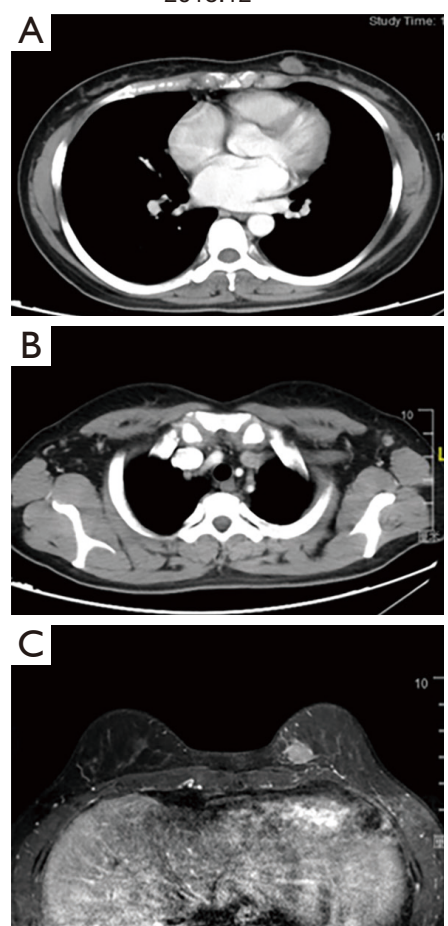

2019.3
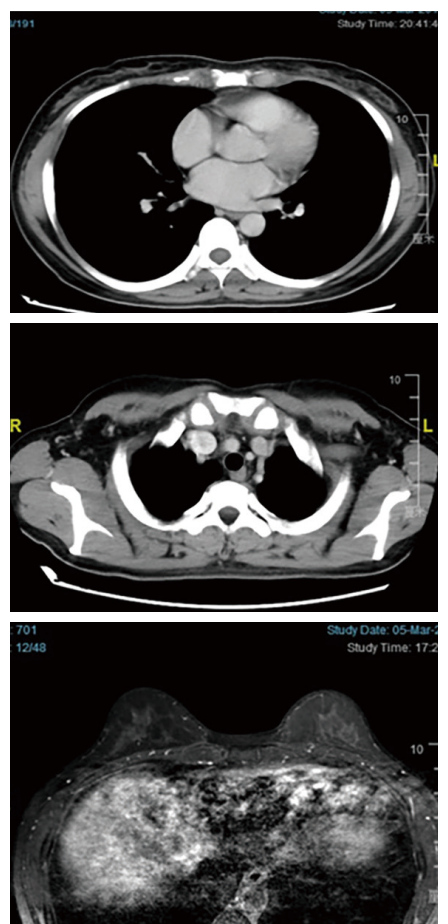

2020.10
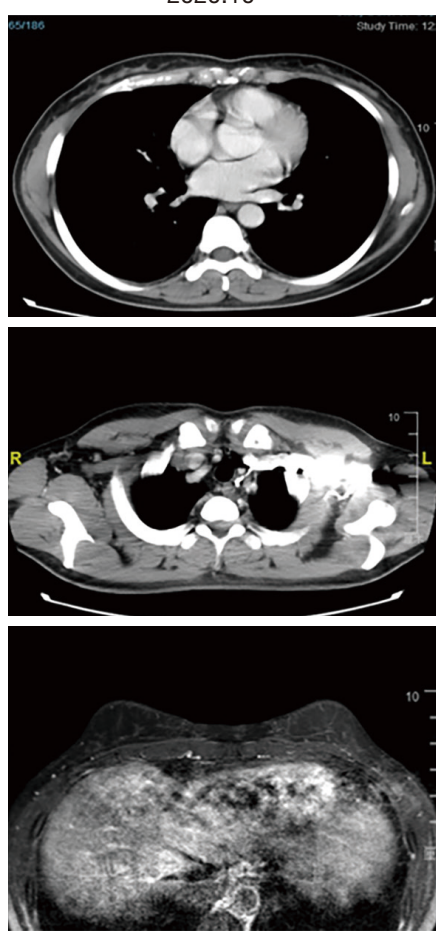

2021.3
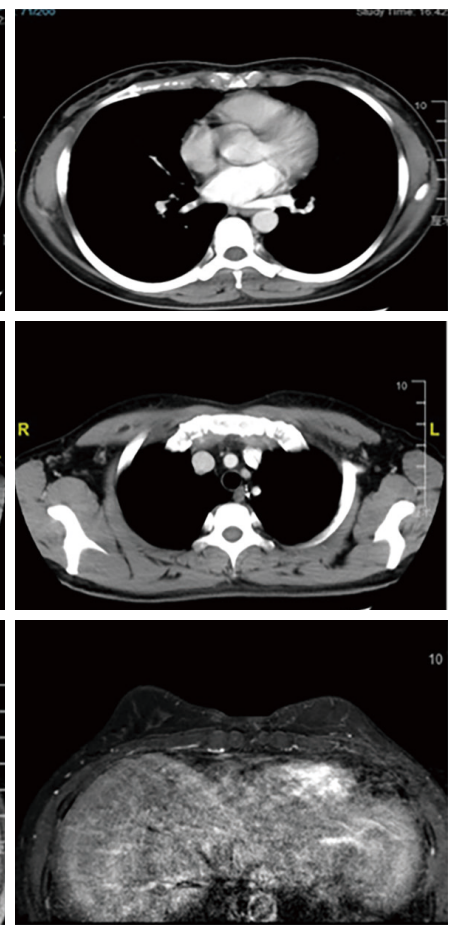

Figure 4 The efficacy of pyrotinib and capecitabine as shown by CT (A,B) and MRI (C) after the occurrence of primary trastuzumab resistance. CT, computed tomography; MRI, magnetic resonance imaging.

images.

\section{Discussion}

The patient was diagnosed with invasive left breast carcinoma accompanied with bilateral axillary and ipsilateral supraclavicular lymph node metastases. CAM is considered to be stage IV, but it is different from other common distant metastases that require local therapy, including metastasis of the liver, lung, bone, etc. There are no clear guidelines regarding the therapeutic management; therefore, individualized therapy should be considered.

Although contralateral axillary dissection can provide excellent axillary management, CAM is associated with systemic metastasis, and locally advanced and aggressive tumors should be considered along with a good possibility of future distant metastasis to the brain, liver, bone, and lung (5). Even though mastectomy is not recommended due to the small likelihood of contralateral occult breast cancer, systemic therapy should be considered. There is some controversy about whether local management is needed for breast cancer patients with stage IV metastasis.
Several different clinical trials, including the MF07-01 (6) and E2 108 trials presented at the 2020 American Society of Clinical Oncology (ASCO) meeting, report varied results regarding local surgical treatment in the diagnosis of de novo Stage IV breast cancer. Therefore, there is no standard guideline in cases of metastatic breast cancer. The axillary lymph node metastasis is usually determined mainly through physical examination, B-ultrasound, CT, and breast MRI, and if necessary, PET-CT. According to the imaging data and physical examination, a thick needle aspiration biopsy will be performed to clarify the pathology and immunohistochemistry. However, the imaging data, including PET-CT, didn't give a definite indication for the patient. For patients considering axillary lymph node metastasis, as it will mainly be used for diagnosis, a thick needle puncture will be sufficient and surgical biopsy has little value. For patients with clinical stages $\mathrm{cN} 1$ and $\mathrm{cN} 2$, after neoadjuvant treatment, in addition to the treatment of primary breast lesions, the axillary lymph nodes will be cleaned, so there is no need to perform surgery when determining whether there is lymph node metastasis; for patients with other distant patients metastasis, even 
if there was axillary lymph node metastasis, surgery is usually not considered necessarily. In our opinion, CAM is considered as a distant metastasis, and thus treating the patient palliatively is too simplistic. In our case, the patient was unwilling to undergo surgery and showed no risk of locoregional ulceration in the breast mass; therefore, she was provided with systemic therapy.

Since pertuzumab had not been approved in China at the time when the patient was diagnosed with HER-2 positive advanced breast cancer. We selected trastuzumab as a single anti-HER-2 target therapy in the first-line treatment. Through the treatment, the patient was sensitive to anthracyclines combined with cyclophosphamides for 4 cycles, and the mass reduced over $50 \%$ in size in the left breast tumor and ipsilateral axillary lymph node; the evaluation of therapeutic effect was partial response (PR). Then, the patient received treatment of trastuzumab combined with docetaxel. The mass in the breast and axillary lymph nodes did not shrink further during the trastuzumab-based treatment. The status of this patient's tumor was ER- and PR+, which is very rare in clinic, with patients usually being hormone receptor positive $(\mathrm{HR}+)$ when they are retested (7). Therefore, we selected trastuzumab combined with endocrine therapy as the maintenance treatment.

However, the PFS for the trastuzumab treatment was only 5 months, likely due to primary trastuzumab resistance (8). The NCCN guidelines, as informed by the EMILIA clinical trial (9), recommend trastuzumab-DM1 (T-DM1) as the standard treatment for progression after trastuzumab, as it typically yields a PFS of 9.6 months. However, T-DM1 has not yet been approved in advanced HER-2-positive breast cancer patients in China by the CFDA. However, lapatinib combined with capecitabine has been approved as the standard second-line treatment of HER-2-positive MBC. According to the result of multicentered, randomized phase II and III clinical trials, pyrotinib and capecitabine treatment confer greater clinical benefits than do lapatinib and capecitabine treatment in patients with HER-2-positive MBC. In these trials, the PFS for pyrotinib treatment was nearly 12 months compared to 6.4 months for lapatinib $(10,11)$. We selected the second-line treatment of pyrotinib combined with capecitabine for this patient: the clinical evaluation was a near CCR and a PFS of over 27 months. Thus far, no data exist comparing the effect of pyrotinib combined with capecitabine versus T-DM1 which treatment is better and this could be an avenue of further research. During the treatment, the main side effects in the first 2 cycles were grade 2 diarrhea, which was be alleviated by loperamide, and grade 1 hand-foot syndrome, which was managed by symptomatic treatment.

In conclusion, pyrotinib can be considered as a reasonable option for patients with primary trastuzumab resistance, and systemic treatment may be the preferred treatment mode for breast cancer with CAM.

\section{Acknowledgments}

Funding: This study received funding from the Jiangsu Provincial Medical Youth Talent (No. QNRC2016647).

\section{Footnote}

Reporting Checklist: The authors have completed the CARE reporting checklist. Available at https://dx.doi. org/10.21037/apm-21-1364

Conflicts of Interest: All authors have completed the ICMJE uniform disclosure form (available at https://dx.doi. org/10.21037/apm-21-1364). The authors have no conflicts of interest to declare.

Ethical Statement: The authors are accountable for all aspects of the work in ensuring that questions related to the accuracy or integrity of any part of the work are appropriately investigated and resolved. Written informed consent was obtained from the patient for publication of this case report and any accompanying images. All procedures performed in studies involving human participants were in accordance with the ethical standards of the institutional and/or national research committee(s) and with the Helsinki Declaration (as revised in 2013).

Open Access Statement: This is an Open Access article distributed in accordance with the Creative Commons Attribution-NonCommercial-NoDerivs 4.0 International License (CC BY-NC-ND 4.0), which permits the noncommercial replication and distribution of the article with the strict proviso that no changes or edits are made and the original work is properly cited (including links to both the formal publication through the relevant DOI and the license). See: https://creativecommons.org/licenses/by-ncnd/4.0/. 


\section{References}

1. Devitt JE, Michalchuk AW. Significance of contralateral axillary metastases in carcinoma of the breast. Can J Surg 1969;12:178-80.

2. Zhu Y, Li L, Zhang G, et al. Metabolic characterization of pyrotinib in humans by ultra-performance liquid chromatography/quadrupole time-of-flight mass spectrometry. J Chromatogr B Analyt Technol Biomed Life Sci 2016;1033-1034:117-27.

3. Li X, Yang C, Wan H, et al. Discovery and development of pyrotinib: A novel irreversible EGFR/HER2 dual tyrosine kinase inhibitor with favorable safety profiles for the treatment of breast cancer. Eur J Pharm Sci 2017;110:51-61.

4. Blair HA. Pyrotinib: First Global Approval. Drugs 2018;78:1751-5.

5. Takahata K, Horiuchi S, Tadokoro Y, et al. Oxytocin levels in low-risk primiparas following breast stimulation for spontaneous onset of labor: a quasi-experimental study. BMC Pregnancy Childbirth 2019;19:351.

6. Soran A, Ozmen V, Ozbas S, et al. Randomized Trial Comparing Resection of Primary Tumor with No Surgery in Stage IV Breast Cancer at Presentation: Protocol MF07-01. Ann Surg Oncol 2018;25:3141-9.

Cite this article as: Yuan Y, Hu S, Qian Y, Zhang L. The successful application of pyrotinib in the treatment of primary trastuzumab-resistant HER-2-positive breast cancer with bilateral axillary lymph node metastasis: a case report. Ann Palliat Med 2021;10(6):7138-7145. doi: 10.21037/apm-21-1364
7. Kiani J, Khan A, Khawar H, et al. Estrogen receptor alphanegative and progesterone receptor-positive breast cancer: lab error or real entity?. Pathol Oncol Res 2006;12:223-7.

8. Harbeck N, Huang CS, Hurvitz S, et al. Afatinib plus vinorelbine versus trastuzumab plus vinorelbine in patients with HER2-overexpressing metastatic breast cancer who had progressed on one previous trastuzumab treatment (LUX-Breast 1): an open-label, randomised, phase 3 trial. Lancet Oncol 2016;17:357-66.

9. Verma S, Miles D, Gianni L, et al. Trastuzumab emtansine for HER2-positive advanced breast cancer. N Engl J Med 2012;367:1783-91.

10. Ma F, Ouyang Q, Li W, et al. Pyrotinib or Lapatinib Combined With Capecitabine in HER2-Positive Metastatic Breast Cancer With Prior Taxanes, Anthracyclines, and/or Trastuzumab: A Randomized, Phase II Study. J Clin Oncol 2019;37:2610-9.

11. Yan M, Bian L, Hu X, et al. Pyrotinib plus capecitabine for human epidermal growth factor receptor 2-positive metastatic breast cancer after trastuzumab and taxanes (PHENIX): a randomized, double-blind, placebocontrolled phase 3 study. Transl Breast Cancer Res 2020;1:13.

(English Language Editor: J. Gray) 